\title{
Experiential Simulation and Psychological assessment as a learning process in architectural higher education
}

\author{
Barbara E. A. Piga ${ }^{1, *}$, Nicola Rainiso ${ }^{2}$, and Marco Boffi ${ }^{2}$ \\ ${ }^{1}$ Laboratorio di Simulazione Urbana fausto Curti, Dipartimento di Architettura e Studi Urbani, \\ Politecnico di Milano, Milan, Italy \\ ${ }^{2}$ Dipartimento di Beni Culturali e Ambientali, Università degli Studi di Milano, Milan, Italy
}

\begin{abstract}
This paper presents the advancements of an applied methodology developed by the authors, namely an architect and two environmental psychologists, based on Virtual Reality coupled with psychological surveys - developed ad hoc by the authors - for urban design; in particular, the paper discusses its application in the field of architectural higher education. The proposed method is based on the pre-evaluation of people's urban experience for testing the effectiveness of design solutions, discovering the unforeseen, and reducing the risk of failure. The method connects architectural to psychological approaches and investigations. The procedure can be schematized as follows: (1) final users navigate the simulation of the design scheme and feedback is collected; (2) the analysis of these collected data is run by professionals and discussed with the designers that then develop the final design solution. In this contribution, we illustrate the application to the urban design development carried out by 100 students of the last year of the Master of Science in Architecture and Urban Planning working in groups. The paper will firstly synthetically present the overall research; secondly, it will argue about its application for higher education; finally, it will conclude by describing the pros and cons of the experimentation, especially in relation to the whole learning and maieutic process and its impact on the design solutions adopted by students, with a focus on the experiential multisensory design.
\end{abstract}

\section{Research framework, methodology and case study application}

This contribution presents the educational effects of a design methodology developed by the authors that links experiential simulation, i.e. Virtual Reality navigated with Head Mounted Displays (HMD), with psychological investigations, i.e. a questionnaire for assessing the experience in a given space (physical or virtual). The approach and the methodology are part of a wider research under development $[1,2,3,4]$. The broad goal of such approach is to

\footnotetext{
* Corresponding author: barbara.piga@polimi.it
} 
increase the awareness of designers about the impact of their design projects on people's experience, relying on multidisciplinary tools and contextual data collection to inform the designers. Using concepts and methodologies derived from social sciences in general, with a specific focus on psychology in this case, and tools such as Virtual Reality, it is possible to investigate and get a picture of the social, cultural, symbolic context induced by design projects before construction. To reach this goal, the authors developed a tool apt to collect quantitative and qualitative data about the experience of people in the environment, that can be used both in currently accessible spaces (physical condition) and not accessible/future spaces (virtual condition). The expectation is to provide the designers with more sources of inspiration for their design process, and to support their creativity in answering people's needs. In this paper, the focus is on the application of the above-mentioned methodology in higher architectural and urban planning education, namely in the course of Architectural and Urban Simulation lead by prof. B. Piga \& R. Salerno, that is addressed to students of the Master of Science. The presented learning methodology was applied for two years, involving a total number of 100 students divided in 14 groups.

The learning methodology is based on a combination of architectural and psychological approaches for teaching directed to the same goal, namely, the anticipation of inhabitants' urban experience leads by design projects. The method is a combination of theoretical lectures (with two main subjects: simulations for urban design and environmental psychology) and practical exercises. Most of theoretical lectures regard the possible application of different kinds of simulations for urban design; the main goal is to build a critical capability to evaluate the proper tools in order develop and test design proposals paying attention to people's experience $[5,6,7]$. Specific lectures are instead related to some key concepts in the field of environmental psychology, with the goal to discuss some basic disciplinary concepts with students who, in most of the cases, are facing the subject for the first time. Among the issues presented to explore the features of the interaction between people and environment, a key role is devoted to the Preference Matrix, explaining environmental preferences through four main concepts [8]; a second main topic is instead the Flow Theory [9], which explains how people build their own wellbeing when involved in engaging activities and how this can have an impact on the relationship with the environment [2]. While presenting the specific topics, teachers refer to the other discipline in order to clarify the strong link between the two: the theoretical background is in fact structured in order to deliver a comprehensive educational message regarding the human/environment relationship. The practical application of the method consists in two main phases; the first one is related to the development of the design project and its representation and is articulated in a series of interrelated exercises [1]; the second phase consists in showing inhabitants the produced simulation, and subsequently administering an ad hoc questionnaire. The first phase must necessarily start before the second one and finish after that, it is indeed a continuous and long process.

The entire process aims at developing and testing the effectiveness of the design project. To do that, the procedure makes use of experiential simulation visualized immersively with simple and low-cost Head Mounted Displays (CardBoards). This navigation of the transformed environment was firstly used for training students to envision the subjective experience generated by their design schemes; the recursive procedure of passing from bidimensional to tri-dimensional representation along with an immersive simulation of the environment supported participants to connect the geometrical representations to the perceptual dynamic experience, i.e. to develop the ability to read abstract depictions of the urban layout and interpret it as subjective navigation of the environment. This is a crucial learning tool that facilitates the development of the typical architects' ability of encoding and 
correctly understanding technical representation [10]. Secondly, students are asked to fill in the questionnaire that investigates some psychological constructs in order to collect their design desiderata, i.e. their expectations in terms of people reactions to their design solution. After this, they are trained to administer the same survey to inhabitants that have virtually navigated their proposal. They are supposed to get around fifty respondents in order to complete the procedure. Later, the environmental psychologists analyze the collected data, without having seen the projects, and they present the outcomes of the study to students. The discussion of results is done in a collegial way, firstly by presenting the overall outcomes of all groups in a comparative way, and secondly by commenting group by group. In this phase, the interaction with the professors of the architectural field is of course very high in order to facilitate students in reading the relationship between the psychological reaction of people and the design of the physical environment. Students actively participate in this dialogue, providing their comments or posing questions. Some general comments on the methodology and the entire process are collected during this phase. After the discussion of results, students have to send a short text commenting the experience. Their feedback is relevant to refine the entire procedure. A process of systematization of results allows to organize some relevant key points emerged from the interaction with students, as described below. The closing of the course is devoted to the fine tuning of the design project considering the survey outcomes. Ideally, it should be a recursive process applying the testing procedure with final users until the results are satisfying; of course, due to time constraints this last process is hardly achieved.

As mentioned above, with the aim of deepening the teaching/educational potential of the procedure, students were asked to provide detailed feedback on the impact of the tools used on their ability to think/rethink the project and on the application of tools and procedure with the participants involved in the evaluation process. The collected observations highlight some emerging issues that can be organized in three macro-categories, discussed in the following paragraphs.

\subsection{Impacts on design thinking}

Under this umbrella, it is possible to collect all feedback related to the different ways in which students' design thinking has been "triggered" by the proposed methodology. In fact, students agree with each other in stating that the process had a significant impact on their design thinking, by enriching and improving their usual approach and contributing to the final solution. They affirm that this improvement process is twofold, as the increase in creativity is connected both with the quality and the quantity of information. On the one hand, through the questionnaire they gained access to information already taken into account during the design phase but represented and communicated in a more extensive way thanks to the visualization of questionnaire outcomes (Fig. 01). On the other hand, they appreciate the emergence of data/information not originally foreseen, that lead to a general increase of the design quality. To encourage their creativity, the direct confrontation with future users is perceived as crucial, since it has been an absolute novelty for them. Finally, among the elements that brought them to modify or fine tune their design project students mentioned:

- a more systemic perspective, given by the environmental perception as a psychological totality by the users, that empowered their abilities in thinking about the general relationships between spatial elements;

- the acquisition of a further professional point of view on space, mainly focused on the emotional and experiential aspects; 
- a new practical ability related to the stronger and more evident connections between the physical and psychological environmental dimensions, that produced a more conscious and adequate response to users' needs;

- possibility of "measuring" the symbolic and iconic strength of their original idea.

\subsection{Practical ways for implementing the project and interacting with the psychological data}

This category collects the main feedback related to how the students have modified their design projects after receiving the feedback from the teachers, both architects and psychologists. Obviously, different intervention modalities were applied depending on the feedback. A basic modality to explain students the gap between their desiderata and users' perception was based on the direct comparison between these data: this procedure enables us to highlight relevant mismatches. From this shared starting point, different perspectives in redefining/redesigning the design project have been adopted. These can be roughly summed up as follows:

- extensive redesign of the project;

- modification of some variables, not only when evaluated as critical by users but also when not consistent with the general concept;

- addition of some visual elements or furniture to reinforce some spatial features/connotations;

- implementation of the simulation's technical quality, since some misunderstanding by users was ascribable to the lack or low quality of the simulation features (e.g. colors, sharpness, people etc.).

\subsection{Features characterizing the tool itself in interacting with the audience}

Students state that using a VR CardBoard had a direct impact on the audience, who was immediately involved and interested in interacting. HMDs seems to be an ideal tool to encourage a playful approach that support citizens' engagement in the participation process. They also stressed that this may be a risk to the results, as people tend to be more interested in the medium than in the design content. On the contrary, many participants stressed that the survey questions appear boring and repetitive. 


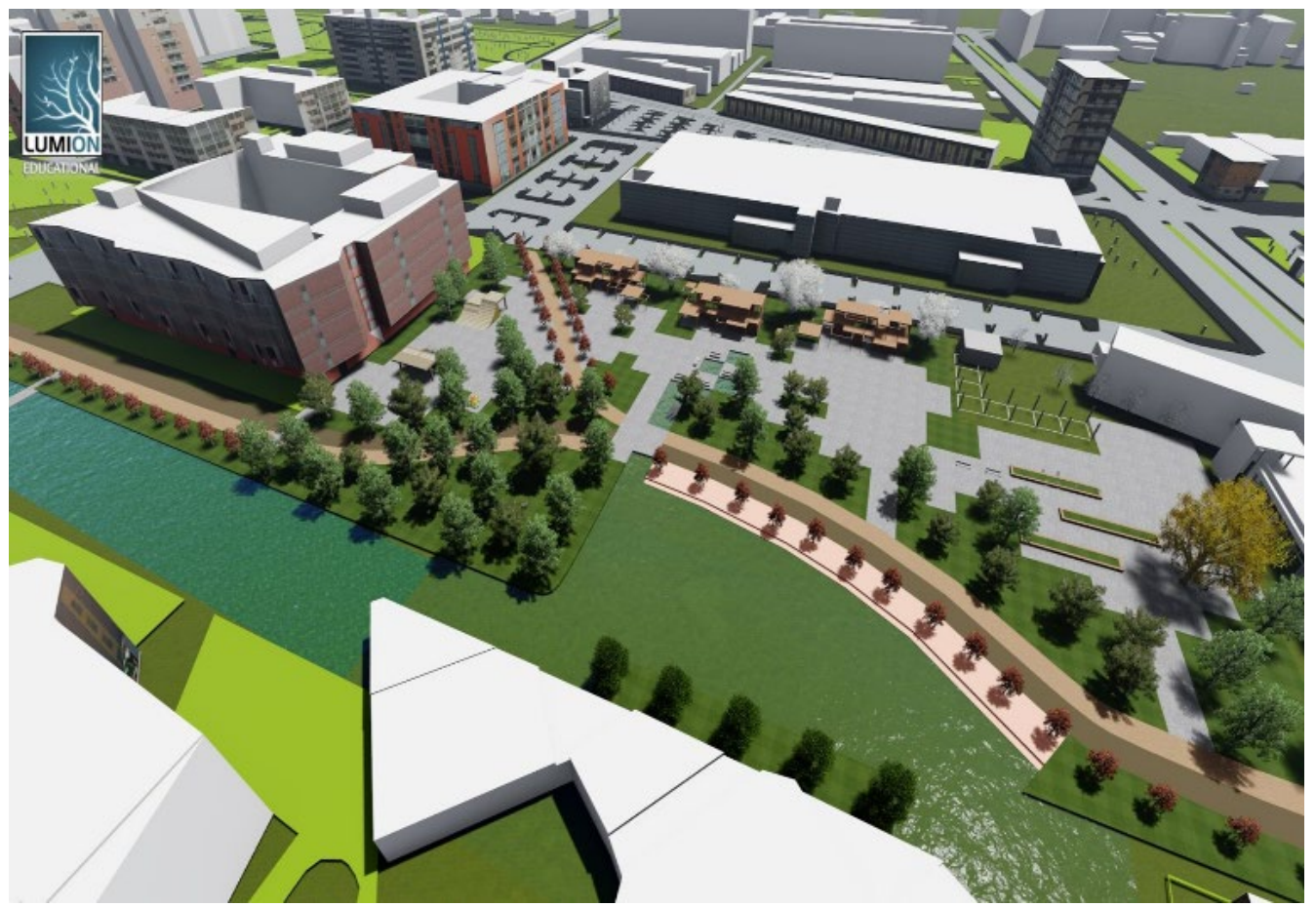

Fig. 1. Bird's eye view of the preliminary design proposal "City Park" by one group of students (above); and one example of outcomes of the psychological assessment: the activities with the red square are the ones. The final design project at the end of the process can be view at: https://youtu.be/QG9t1R6j-Is (students credits: M. Ainsa, H. Erdin, M. Ghedamsi, A. Ginter, E. Hjorstam, I. Kovacevic, P. Kwiatkowska, E. Le Helloco).

\section{Conclusions}

In conclusion, it is possible to highlight that the entire process acts as a learning tool for students. In general, it is possible to understand from their comments that the method enables them to better envision and focus the experiential outcomes of their design project, and therefore its civic relevance. At the same time, the process allows students to deal with an interdisciplinary approach and to experiment first-hand the relevance of disciplinary collaboration. The role of representation, both on the side of simulation for design thinking and on the side of communication of design project, that permit to collect users' feedback, is crucial for the entire process. The use of HMDs, and specifically CardBoards, was relevant for three main reasons: (i) these provide a one-on-one scale interaction with the design project, that was traditionally unusual in the architectural field, enabling to virtually test the environment in a subjective way; (ii) participants to the survey are generally intrigued by the tool and engaged by the experience, and this is generally facilitating designers and interviewers in running the process; (iii) even if the quality of the simulation is not that high using CardBoards and standard smartphones, due to the affordable cost of the devices it is possible to run several virtual navigations in parallel; this last point contributes to the replicability of the setting in university courses or in the professional practice, even in small offices. Of course, the support of professionals for training the interviewers, performing the analysis, and interpreting the outcomes is in any case necessary. Regarding the trustability of such devices for these types of investigations, a recent research developed by the author 
(under publication) demonstrates that even this low-cost device enables a well-balanced quivalence of responses between the simulated and the real environment.

\section{References}

1. Piga, B. E. A. (2017). Experiential Simulation for Urban Design: From Design Thinking to Final Presentation. In B. E. A. Piga \& R. Salerno (Eds.), Urban Design and Representation. A Multidisciplinary and Multisensory Approach (pagg. 23-36). Springer International Publishing.

2. Boffi, M., \& Rainisio, N. (2017). To Be There, Or Not To Be. Designing Subjective Urban Experiences. In B. E. A. Piga \& R. Salerno (Eds.), Urban Design and Representation. A Multidisciplinary and Multisensory Approach (pagg. 37-53). Springer International Publishing.

3. Piga, B. E. A., Morello, E., Boffi, M., Rainisio, N., \& Di Liberto, G. (2017). Sharing the experience of urban design schemes through immersive simulation. In T. Maver, P. Chapman, C. Platt, V. Portela, \& D. Eaton (Eds.) (pagg. 244-251). Envisioning Architecture: Space / Time / Meaning, 6-9/09/2017, Glasgow (GB): Mackintosh School of Architecture and the School of Simulation and Visualization at the Glasgow School of Art.

4. Piga, B. E. A., Boffi, M., \& Rainisio, N. (2017). Experiential Simulation and Environmental Psychology for Anticipating Users' Reactions to Design Projects: An Application in Architectural Higher Education. Proceedings of the International and Interdisciplinary Conference IMMAGINI? Image and Imagination between Representation, Communication, Education and Psychology, 1(9:968), 1-9

5. Rasmussen, S. E. (1964). Experiencing Architecture (originally published in 1959). Cambridge MA: MIT Press.

6. Bosselmann, P. (1998). Representation of Places: Reality and Realism in City Design. Berkeley, Los Angeles, London: University of California Press.

7. Carmona, M., Tiesdell, S., Heath, T., \& Oc, T. (2010). Public Places, Urban Spaces: The Dimensions of Urban Design. Routledge.

8. Kaplan, R., \& Kaplan, S. (1989). The Experience of Nature: A Psychological Perspective. Cambridge University Press: New York, NY, USA.

9. Csikszentmihalyi, M. (1975). Beyond Boredom and Anxiety. Jossey-Bass: San Francisco, CA, USA.

10. Schön, D. A. (1987). Educating the Reflective Practitioner: Toward a New Design for Teaching and Learning in the Professions. Wiley. 